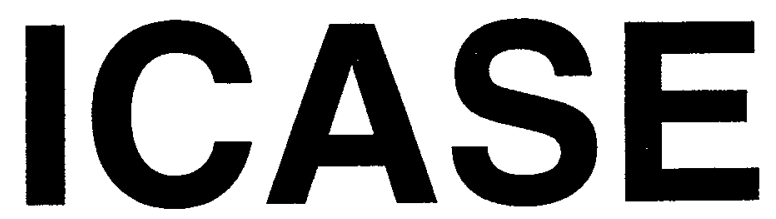

\title{
OPTIMUM SUCTION DISTRIBUTION FOR TRANSITION CONTROL
}

\section{P. Balakumar \\ P. Hall}

NASA Contract No. NASI-19480

December 1996

Institute for Computer Applications in Science and Engineering NASA Langley Research Center

Hampton, VA 23681-0001

Operated by Universities Space Research Association

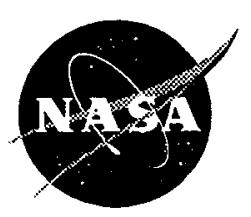

National Aeronautics and Space Administration

Langley Research Center

Hampton, Virginia 23681-0001 


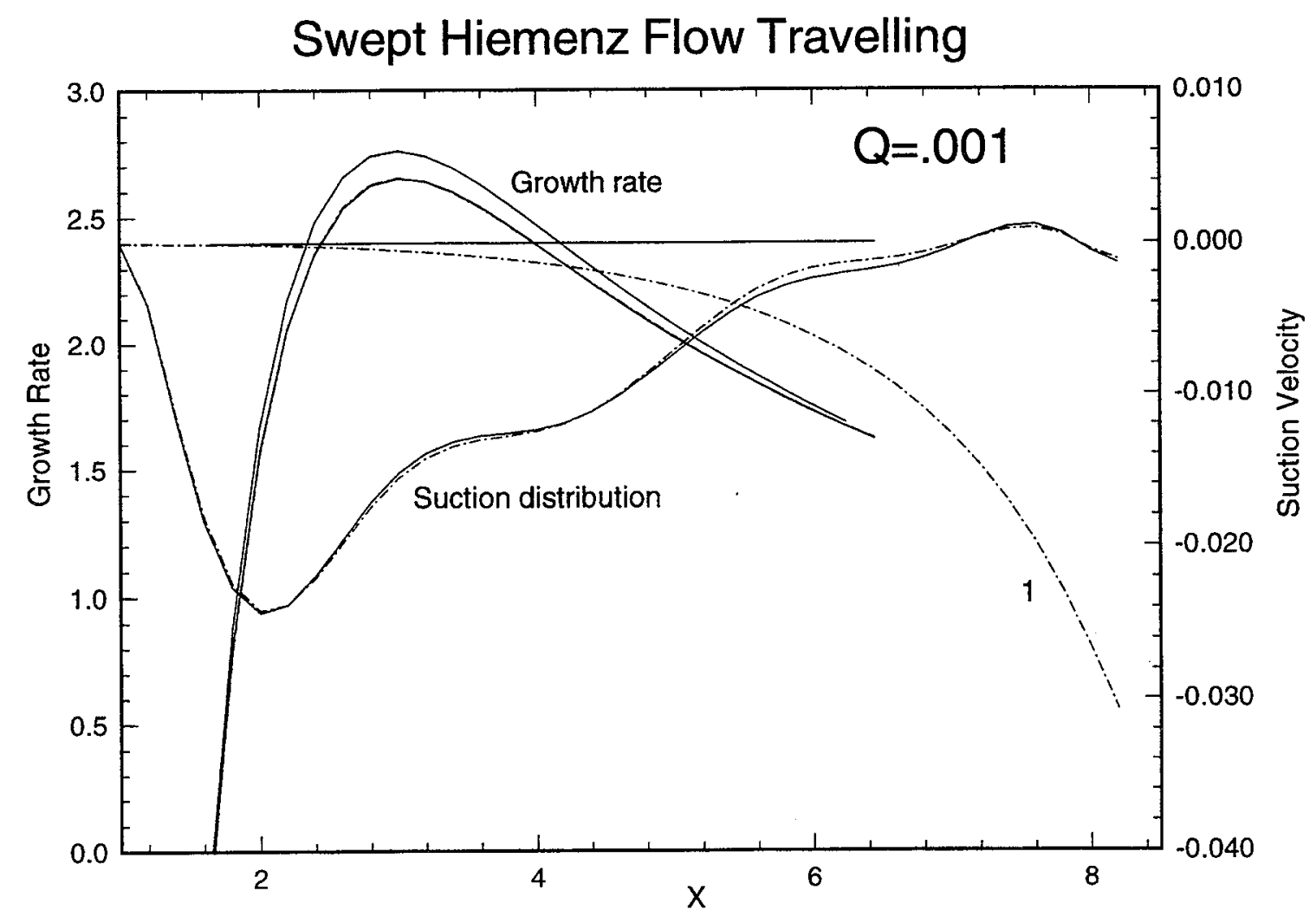

Figure 9. Convergence towards the optimum suction distribution and the growth rate for the swept Hiemenz flow. $\bar{R}=500, \mathrm{Q}=.001, \mathrm{~N}=8$. 


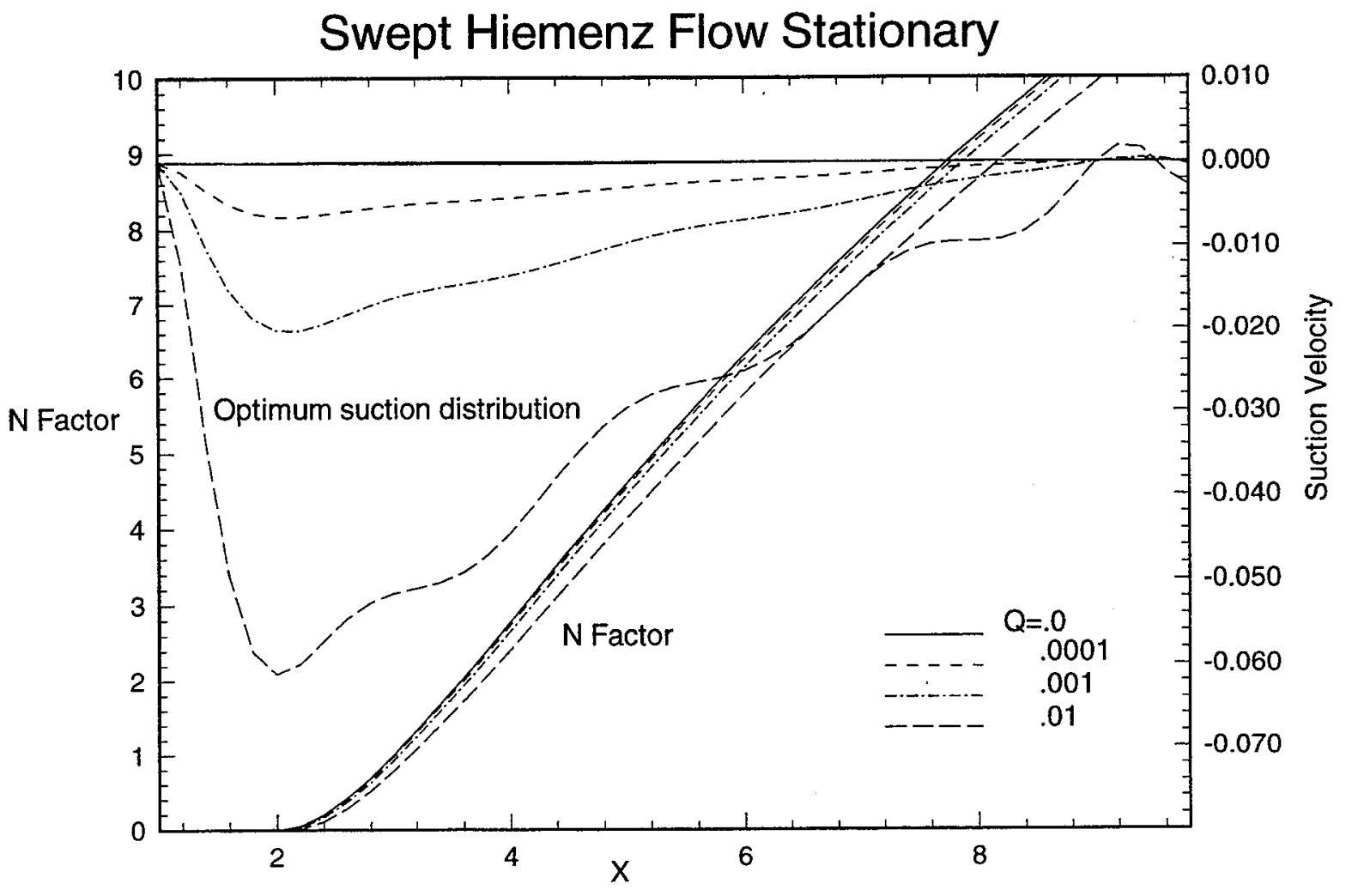

Figure 11. Optimum suction distribution and the $\mathrm{N}$-factor for the swept Hiemenz flow for stationary disturbances. $\bar{R}=500, \mathrm{~N}=10$. 


\section{Swept Hiemenz Flow}

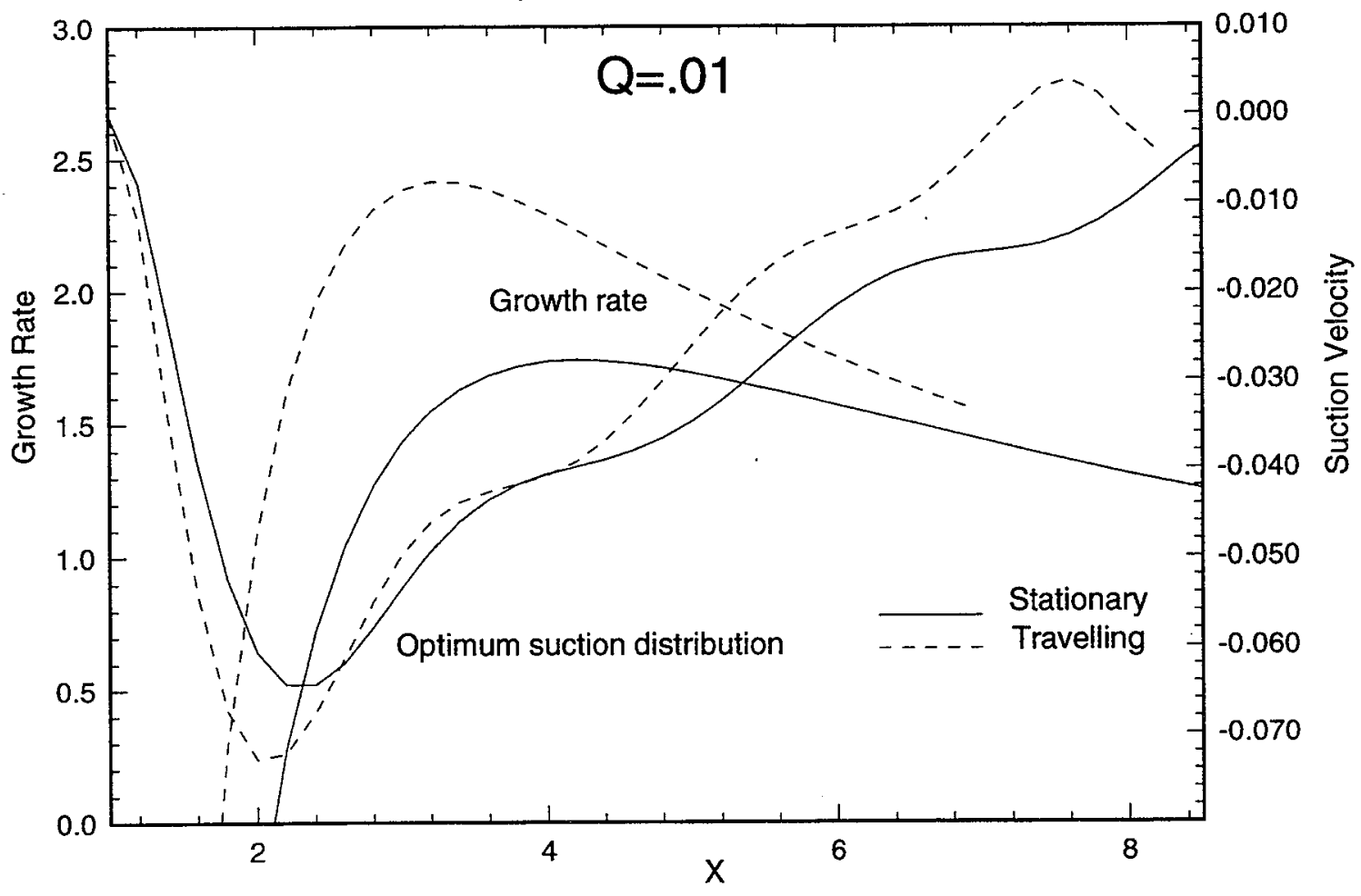

Figure 13. Optimum suction distribution and the $\mathrm{N}$-factor for the stationary and travelling disturbances. $\mathrm{Q}=.01, \mathrm{~N}=8$. 


\title{
OPTIMUM SUCTION DISTRIBUTION FOR TRANSITION CONTROL*
}

\author{
P. Balakumar* \\ Department of Aerospace Engineering \\ Old Dominion University \\ Norfolk, Virginia 23529 \\ P. Hall $\dagger$ \\ Department of Mathematics \\ Imperial College \\ London, UK
}

\begin{abstract}
The optimum suction distribution which gives the longest laminar region for a given total suction is computed. The goal here is to provide the designer with a method to find the best suction distribution subject to some overall constraint applied to the suction. We formulate the problem using the Lagrangian multiplier method with constraints. The resulting non-linear system of equations is solved using the Newton-Raphson technique. The computations are performed for a Blasius boundary layer on a flat-plate and crossflow cases. For the Blasius boundary layer, the optimum suction distribution peaks upstream of the maximum growth rate region and remains flat in the middle before it decreases to zero at the end of the transition point. For the stationary and travelling crossflow instability, the optimum suction peaks upstream of the maximum growth rate region and decreases gradually to zero.
\end{abstract}

This work was supported by the National Aeronautics and Space Administration under NASA Contract No. NAS1-19480 while the authors were in residence at the Institute for Computer Applications in Science and Engineering (ICASE), NASA Langley Research Center, Hampton, VA 23681-0001. 


\section{Introduction}

There exists several methods to control the laminar boundary layers, e.g. suction, cooling the wall, modifying the shape, artificial blowing etc. The main purposes of these controls are to: (1) avoid the laminar separation and/or (2) delay or prevent the transition from laminar to turbulent flow in the laminar boundary layers and hence reduce the drag force and/or increase the lift. In this paper we are concerned with the use of suction to control transition in laminar boundary layers.

The effects of suction on a laminar boundary layer are to reduce the thickness of the boundary layer and to make the boundary layer becomes fuller near the wall. Since the viscous instability is directly related to the second derivative of the streamwise velocity at the wall the boundary layers with suction becomes more stable than that without suction. In flows with adverse pressure gradients the suction removes or weakens the inflection in the velocity profile and hence inhibits the inviscid instability.

In theory with enough amount of suction, it is possible to completely prevent the transition from laminar to turbulent flow. However, increasing the amount of suction has two adverse consequences. Firstly to apply a large amount of suction one needs to drive a big pump and hence the saving in drag due to suction will be offset by the energy needed for the pump. Secondly, when the suction is applied the boundary layer becomes thin and hence the skin friction increases. It is therefore important to keep the amount of suction required to a minimum. The questions to ask are what is the minimum amount of suction required to satisfy a design requirement and what is the best suction distribution? In this work we investigate these questions.

The use of suction as a feasible and effective method to control laminar boundary layers existed from the era of Prandtl. Pfenninger (1977) summarizes the research work done at Northrop about using suction to control transition in laminar boundary layers. It has been verified theoretically and experimentally that the boundary layer suction prevents laminar separation and transition in flows with any pressure rise at high Reynolds numbers. Reed and Nayfeh (1986) investigated the effects of different suction strip configurations on the stability using linear triple-deck theory and influence coefficient method. The change in the integrated growth rate at a fixed location is obtained as a linear combination of suction strip velocities. The optimum location of the suction strip is determined by the point where the multiplying constant takes the maximum value. Their numerical results showed that the coefficient becomes maximum near the branch 1 and branch 2 neutral points and near the former being larger. This showed that suction is more effective when placed near the branch 1 neutral point. In a companion paper Reynolds and Saric (1986) investigated experimentally the effect of different suction strip configuration on the amplification of the disturbances. They used two suction panels each consists of 15 suction strips. Their experimental findings also agreed with the theoretical results that the suction be concentrated near the neutral point region to obtain the largest reduction in the integrated growth. 
The state of the art transition prediction method that is used in the design is the empirical $\mathrm{e}^{\mathrm{N}}$ method. The numerical procedure to locate the transition onset involves two steps. The first step is to compute the mean flow for a given suction distribution, and the second step is to locate the transition onset using the linear stability and the $\mathrm{e}^{\mathrm{N}}$ method.

To find the optimum suction distribution, trial and error procedures are commonly used. In those methods, a few different suction distributions are tried, and the distribution which gives the furthest transition onset is selected as the design suction distribution. In this work, we develop a method based on the Lagrangian multipliers technique, to find the optimum suction distribution for a given suction constraint which is dictated by the designer.

\section{Formulation}

We are concerned with finding the optimum suction distribution to stabilize a laminar boundary layer as much as possible within some ovearall constraint on the amount of suction to be used. The wall suction basically modifies the mean velocity profiles inside the boundary layer, and these changes in the mean flow profiles influence the stability characteristics of the boundary layer. The transition from laminar to turbulence in a low disturbance environment is directly related to the stability characteristics of the boundary layer profiles. Hence the transition point is connected to the suction through the stability and the mean flow profiles. Thus the analysis to control and/or predict the transition onset point involves the following three steps and we describe them each in the subsequent sections:

1. Mean flow calculations

2. Stability computations

3. Finding the optimum solution.

\subsection{Meanflow}

In this work we compute the mean boundary layer profiles by solving the boundary layer equations. The analysis is in fact not at all dependent on the nature of the basic boundary layer flow. Let $\mathrm{x}$ be the coordinate in the axial direction, $\mathrm{y}$ be the coordinate in the direction normal to the surface, and $\mathrm{z}$ be the co-ordinate in the spanwise direction. The boundary layer equations for an incompressible three-dimensional flow are

$$
\begin{gathered}
\frac{\partial U}{\partial x}+\frac{\partial V}{\partial y}=0 \\
U \frac{\partial U}{\partial x}+V \frac{\partial U}{\partial y}=U_{e} \frac{d U_{e}}{d x}+\nu \frac{\partial^{2} U}{\partial y^{2}} \\
U \frac{\partial W}{\partial x}+V \frac{\partial W}{\partial y}=\nu \frac{\partial^{2} W}{\partial y^{2}}
\end{gathered}
$$


The boundary conditions are

$$
U=0, \quad V=V_{o}(x), \quad W=0 \text { at } y=0,
$$

and $\quad U \rightarrow U_{e}, W \rightarrow W_{e}$ at $y \rightarrow \infty$.

Here $U_{e}(x), W_{e}$ are the free-stream velocities in the streamwise and spanwise directions and $V_{o}(x)$ is the suction velocity at the wall.

\subsection{Stability}

The growth of a disturbance is calculated by solving the linear parallel stability equations (Drazin \& Reid 1981). Here we discuss the stability equations for an incompressible flow. In the quasi-parallel linear stability theory, the disturbance quantities are written in normal mode form. If $\mathrm{q}(\mathrm{x}, \mathrm{y}, \mathrm{z})$ is a disturbance flow variable, in normal mode analysis, we write

$$
q(x, y, z)=q(y) e^{i \int \alpha d x+i \beta z-\omega t} .
$$

Here $\alpha, \beta$ are the axial and spanwise wave numbers, $\omega$ is the frequency, and $q(y)$ is the eigenfunction. We substitute this expression into the linearized Navier-Stokes equations and, assuming the flow is parallel in the streamwise direction, we obtain an ordinary differential equation for $q(y)$. This equation, along with the homogeneous boundary conditions at the wall and in the free-stream, forms the eigenvalue problem for the wavenumber $\alpha$ and for the eigenfunction $\mathrm{q}(\mathrm{y})$. The three-dimensional linear stability equations for an incompressible flow are

$$
\begin{aligned}
& \frac{d v}{d y}=-i \alpha u-i \beta w, \\
& \frac{d^{2} u}{d y^{2}}=\left\{i(\alpha U+\beta W-\omega) \operatorname{Re}+\alpha^{2}+\beta^{2}\right\} u+\operatorname{Re} \frac{d U}{d y} v+i \alpha R e p \\
& \frac{d p}{d y}=-\frac{1}{R e} i \alpha \frac{\partial u}{\partial y}-\frac{i \beta}{R e} \frac{\partial w}{\partial y}-[i(\alpha U+\beta W-\omega) \\
& \left.+\frac{\alpha^{2}}{R e}+\frac{\beta^{2}}{R e}\right] v \\
& \frac{d^{2} w}{d y^{2}}=\left\{i(\alpha U+\beta W-\omega) R e+\alpha^{2}+\beta^{2}\right\} w+i \beta p \\
& +R e \frac{d W}{d y} v .
\end{aligned}
$$

The boundary conditions are

$$
u=0, v=0, w=0 \text { at } y=0,
$$


and $\quad u \rightarrow 0, w \rightarrow 0$ at $y \rightarrow \infty$. Here $u, v, w$ are the velocities in the axial, normal and spanwise directions and $\mathrm{p}$ is pressure, $\alpha$ is a complex wavenumber

$$
\alpha=\alpha_{r}+i \alpha_{i}
$$

and $\left(-\alpha_{i}\right)$ measures the growth of the disturbances in the axial direction. We note for our control problem that the suction $\mathrm{V}_{\mathrm{o}}$ only enters in the stability problem through the mean flow profiles $\mathrm{U}$ and $\mathrm{W}$.

\subsection{Transition Prediction}

The state of the art transition prediction method that is used in the design is the empirical $e^{N}$ method (Smith 1956, Van Ingen 1956) and here we use this method to locate the transition onset point $x_{T}$. In this method, the transition point is defined when the integrated growth rate reaches a prescribed value $\mathrm{N}_{0}$.

$$
\int_{x_{N}}^{x_{T}} \sigma(x) d x=N_{0} .
$$

Here $\sigma$ is the growth rate, $x_{N}$ is the neutral point where $\sigma\left(x_{N}\right)=0$ and $x_{T}$ is the transition onset point.

\subsection{Control and Optimum Suction Distribution}

Any control strategy to stabilize the boundary layer must reduce $\sigma$ and hence increase the laminar region or in other words maximize $x_{T}$. Therefore the problem is to maximize $x_{T}$ for a given constraint on the suction. Let us assume that the suction distribution $V_{o}(x)$ is constrained by

$$
\int_{x_{\min }}^{x_{\max }} V_{o}^{2}(x) d x=Q .
$$

Here we assume $V_{o}(x)=0$ for $x \leq x_{\min }$. In our calculations we take $x_{\min }<x_{N}$ and $x_{m a x}$ is much larger than $x_{T}$. Here we have chosen to constrain the suction based on a measure of the work done to supply the suction rather than simply on $V_{o}(x)$ itself. This prevents the somewhat absurd distributions generated in the latter case where for example large amount of suction in potentially unstable regions are balanced by equally large amounts of blowing in the more stable regions. The control problem is then reduced to maximizing

$$
I=\frac{x_{T}^{2}}{2},
$$

with the following constraints

$$
\int_{x_{\min }}^{x_{\max }} V_{o}^{2} d x=Q
$$


and $x_{T}$ is defined by,

$$
\int_{x_{N}}^{x_{x}} \sigma d x=N_{0} .
$$

To simplify the computations, we reduced the continuous problem in $V_{o}(x)$ to a discrete one by expanding $V_{o}(x)$ in terms of an appropriate set of functions. Thus we write

$$
V_{o}(x)=\sum_{1}^{N} a_{n} F_{n}(x)
$$

and for a given $N$ we find $\left\{a_{n}\right\}$ which maximizes $x_{T}$ subject to the constrains (11-12).

\section{Solution Procedure}

The optimum suction distributions are found using the Lagrangian multiplier technique. Thus we write

$$
\begin{aligned}
F & =\frac{x_{T}^{2}}{2}+\lambda\left\{\int_{x_{N}}^{x_{T}} \sigma d x-N_{o}\right\}+\delta\left\{\int_{x_{\min }}^{x_{\max }} V_{o}^{2} d x-Q\right\} \\
& =F\left(x_{T}, \lambda, \delta, a_{i}, \omega, \beta\right) .
\end{aligned}
$$

Here $\lambda, \delta$ are Lagrangian multipliers and $\omega, \beta$ are the frequency and spanwise wavenumber. By differentiating $F$ respect to each variables we obtain the following Euler-Lagrange equations.

$$
\begin{aligned}
& x_{T}+\lambda \sigma\left(x_{T}, a_{i}, \omega, \beta\right)=0, \\
& \int_{x_{N}}^{x_{T}} \sigma\left(a_{i}, \omega, \beta, x\right) d x-N_{o}=0, \\
& \int_{x_{\min }}^{x_{\max }} V_{o}^{2} d x-Q=0, \\
& \lambda \int_{x_{N}}^{x_{T}} \frac{\partial \sigma}{\partial a_{i}} d x+\delta \int_{x_{\min }}^{x_{\max }} \frac{\partial V_{o}^{2}}{\partial a_{i}} d x=0 \quad\{i=1, N\}, \\
& \int_{x_{N}}^{x_{T}} \frac{\partial \sigma}{\partial \beta} d x=0, \\
& \int_{x_{N}}^{x_{T}} \frac{\partial \sigma}{\partial \omega} d x=0 .
\end{aligned}
$$


We note that by including the last two conditions we find the optimum solution in the whole $\beta, \omega$ plane. If we want to find the solution for a fixed quantity of $\beta$, or $\omega$, we remove the appropriate equations from the set eq.(15). In our calculations, we considered two different functions for $F_{n}(x)$, one is a polynomial and the other is a harmonic (sine) function. Thus we have either

$$
V_{o}(x)=\sum_{i=1}^{N} a_{i} X^{i+S}
$$

or

$$
=\sum_{i=1}^{N} a_{i} \sin i \pi X,
$$

where $S$ is an integer which determines the shape of the suction distribution at the initial point $\mathrm{x}_{\mathrm{xmin}}$ and $X=\frac{x-x_{\min }}{x_{\max -x_{\min }}}$. To solve the system, we treat the $\left(a_{i}, \beta, \omega\right)$ as our independent variables and rewrite the equations as

$$
\begin{gathered}
f_{1}=\sum_{k=1}^{N} \sum_{l=1}^{N} a_{k} a_{l} \frac{L}{k+l+2 S+1}-Q_{o}=0, \\
f_{i}=\left(\sum_{j=1}^{N} \frac{a_{j}}{j+2 S+2}\right) \int_{x_{N}}^{x_{T}} \frac{\partial \sigma}{\partial a_{i}} d x- \\
\left(\sum_{j=1}^{N} \frac{a_{j}}{i+j+2 S+1}\right) \int_{x_{N}}^{x_{T}} \frac{\partial \sigma}{\partial a_{1}}=0, \\
f_{N+1}=\int_{x_{N}}^{x_{T}} \frac{\partial \sigma}{\partial \beta} d \beta=0, \\
f_{N+2}=\int_{x_{N}}^{x_{T}} \frac{\partial \sigma}{\partial \omega} d \omega=0 .
\end{gathered}
$$

Therefore we have $(\mathrm{N}+2)$ equations for the $(\mathrm{N}+2)$ variables $\left\{a_{i} i=1, N\right\}, \beta$, and $\omega$. Since this is a nonlinear system it has to be solved iteratively and we used Newton 
linearization procedure which requires the Jacobian of $\left\{f_{i}\right\}$ with respect to the variables $\left\{a_{i}\right\}, \beta$, and $\omega$. For example,

$$
\begin{gathered}
\frac{\partial f_{i}}{\partial a_{k}}=\left(\sum_{j=1}^{N} \frac{a_{j}}{j+2 S+2}\right)\left\{\int_{x_{N}}^{x_{T}} \frac{\partial^{2} \sigma}{\partial a_{i} \partial a_{k}} d x\right. \\
\left.+\frac{\partial x_{T}}{\partial a_{k}} \frac{\partial \sigma}{\partial a_{i}}\left(x_{T}\right)-\frac{\partial x_{N}}{\partial a_{k}} \frac{\partial \sigma}{\partial a_{i}}\left(x_{N}\right)\right\} \\
+\frac{1}{k+2 S+2} \int_{x_{N}}^{x_{T}} \frac{\partial \sigma}{\partial a_{i}} d x \\
-\left(\sum_{j=1}^{N} \frac{a_{j}}{i+j+2 S+1}\right)\left\{\int_{x_{N}}^{x_{T}} \frac{\partial \sigma}{\partial a_{1} \partial a_{k}} d x\right. \\
+\frac{\partial x_{T}}{\partial a_{k}} \frac{\partial \sigma}{\partial a_{1}}\left(x_{T}\right) \\
\left.-\frac{\partial x_{N}}{\partial a_{k}} \frac{\partial \sigma\left(x_{N}\right)}{\partial a_{1}}\right\}-\frac{1}{i+k+2 S+1} \int_{x_{N}}^{x_{T}} \frac{\partial \sigma}{\partial a_{1}} d x . \\
\text { for } i=2, N .
\end{gathered}
$$

Similarly for other $f_{i}$. We observe that to evaluate $\left\{f_{i}\right\}$ and the Jacobian we need the quantities

$$
\begin{array}{lll}
\frac{\partial \sigma}{\partial a_{i}} & \frac{\partial^{2} \sigma}{\partial a_{i} \partial a_{j}} & \\
\frac{\partial \sigma}{\partial \beta} & \frac{\partial^{2} \sigma}{\partial \beta \partial a_{i}} & \frac{\partial^{2} \sigma}{\partial \beta \partial \omega} \\
\frac{\partial \sigma}{\partial \omega} & \frac{\partial^{2} \sigma}{\partial \omega \partial a_{i}} & \\
\frac{\partial x_{T}}{\partial a_{i}} & \frac{\partial x_{T}}{\partial \omega} & \frac{\partial x_{T}}{\partial \beta} \\
\frac{\partial x_{N}}{\partial a_{i}} & \frac{\partial x_{N}}{\partial \omega} & \frac{\partial x_{N}}{\partial \beta}
\end{array}
$$


Amongst these quantities the last two rows can be obtained from the relationships

$$
\begin{gathered}
\frac{\partial x_{T}}{\partial a_{i}}=-\frac{\int_{x_{N}}^{x_{T}} \frac{\partial \sigma}{\partial a_{i}} d x}{\sigma\left(x_{T}\right)} \\
\frac{\partial x_{N}}{\partial a_{i}}=-\frac{\frac{\partial \sigma}{\partial a_{i}}}{\frac{\partial \sigma}{\partial x_{N}}} .
\end{gathered}
$$

and similar forms may be found for the other four quantities in the last two rows. The quantities in the first three rows are obtained by differentiating the stability equations. For example $\frac{\partial \sigma}{\partial a_{i}}$ is obtained from the solution of the system

$$
L\left\{\psi_{i}\right\}=\alpha_{i} q_{1}+q_{2}
$$

where $\mathrm{L}$ is the linear operator eq. (5), $\psi$ is the flow variable $\left\{u, v, w, p^{T}\right\}$ $q_{1}=q_{1}\{\psi\}, q_{2}=q_{2}\left\{\psi, U_{i}, W_{i}\right\}$ and subscript $i$ denotes differentiation respect to $a_{i}$. $\frac{\partial \alpha}{\partial a_{i}}$ can be computed using the adjoint method. We see that this requires the knowledge of the mean flow quantities $\frac{d U}{d a_{i}}$ etc. This is obtained again by differentiating the mean flow equations.

$$
\begin{gathered}
\frac{\partial U_{i}}{\partial x}+\frac{\partial V_{i}}{\partial y}=0 \\
U_{i} \frac{\partial U}{\partial x}+U \frac{\partial U_{i}}{\partial x}+V_{i} \frac{\partial U}{\partial y}+V \frac{\partial U_{i}}{\partial x}=\nu \frac{\partial^{2} U_{i}}{\partial y^{2}} \\
U_{i} \frac{\partial W}{\partial x}+U \frac{\partial W_{i}}{\partial x}+V_{i} \frac{\partial W}{\partial y}+V \frac{\partial W_{i}}{\partial y}=\nu \frac{\partial^{2} W_{i}}{\partial y^{2}}, \\
U_{i}=W_{i}=0 \quad \text { at } y=0, \infty \\
\text { and } \quad V_{i}=X^{i+n} \quad \text { at } y=0 .
\end{gathered}
$$

All the equations are solved using the two-point fourth order compact scheme in the $y$ direction (Malik (1990)) together with a second order accurate method in $x$. 
the suction distribution for the stationary and the travelling disturbances. As expected the travelling disturbances are more unstable than the stationary disturbances. But the maximum suction distribution occurs near the same location for both cases. For the stationary disturbances, the most of the suction is distributed upstream of the maximum amplification rate compared to that for the travelling disturbances. Table 1 shows the variation of the Reynolds number at the transition point $R e_{T}$, the most amplified nondimensional frequency and the most amplified spanwise wavenumber $\beta$ with the suction parameter $\mathrm{Q}$.

\begin{tabular}{|l|c|c|c|c|c|}
\cline { 2 - 6 } \multicolumn{1}{c|}{} & \multicolumn{3}{c|}{ Travelling } & \multicolumn{2}{c|}{ Stationary } \\
\hline $\mathrm{Q}$ & $\mathrm{F}$ & $\beta$ & $\operatorname{Re}_{\mathbf{T}}$ & $\beta$ & $\operatorname{Re}_{\mathbf{T}}$ \\
.0 & $4.4325 \mathrm{E}-3$ & .2988 & 497 & .3327 & 688 \\
.0001 & $4.3881 \mathrm{E}-3$ & .3004 & 503 & .3338 & 691 \\
.001 & $4.2940 \mathrm{E}-3$ & .3037 & 516 & .3370 & 700 \\
.01 & $4.0310 \mathrm{E}-3$ & .3135 & 553 & .3456 & 726 \\
\hline
\end{tabular}

\section{Conclusions}

Optimum suction distribution for transition control is investigated using the Lagrangian multiplier technique with constraints. We imposed a suction constraint based on a measure of the work done to supply the suction. The appropriate constraint should be the real cost to install and operate the suction distribution. Here we basically assumed that this is proportional to the integral of the square of the suction velocity. We investigated the incompressible flow over a flat plate, swept Hiemenz flow.

The converged results are obtained in 4-5 iterations cycles. For the incompressible flow the optimum suction peaks upstream of the most unstable region and remains flat in the middle and becomes zero at the end of the transition. For the travelling and crossflow type instability the suction peaks upstream of the most unstable region and gradually becomes zero at the end.

Another observation was that with increasing suction parameter $Q$, the convergence becomes slower with the polynomial expansion. The sine expansion converges faster but we have to keep a large number of terms to remove the oscillations. However the overall shape, the transition location, growth rate do not change with the increasing number of terms. The amount of suction needed to control the crossflow instability is about two orders of magnitude larger than that for the T-S wave. For example, we can delay the transition on a flat plate by $20 \%$ with $\mathrm{Q}=.001$, and the maximum suction velocity is $\frac{V_{0}}{U_{\infty}}=2 * 10^{-5}$. For the stationary crossflow we delay the transition only by $6 \%$ with $\mathrm{Q}=.01$ and the maximum suction velocity is $\frac{V_{0}}{U_{\infty}}=7 * 10^{-4}$. 


\section{References}

1. Drazin, P. G. \& Reid, W. H. "Hydrodynamic Stability", pp. 216-221, Cambridge University Press, 1981.

2. Pfenninger, W., " USAF \& Navy Sponsored Northrop LFC Research Between 1949 and 1967," AGARD/VKI Special Course on Concepts for Drag Reduction, Rhode-St. Genese, Belgium, 1977.

3. Reed, H. L. \& Nayfeh, A. H. "Numerical-Perturbation Technique for Stability of FlatPlate Boundary Layers with Suction," AIAA Journal, Vol. 24, 1986, pp. 208-214.

4. Reynolds, G. A. and Saric, W. S., "Experiments on the Stability of the Flat-Plate Boundary Layer with Suction," AIAA Journal, Vol. 24, 1986, pp. 202-207.

5. Smith, A. M. O., "Transition, Pressure Gradient and Stability Theory," IX International Congress for Applied Mechanics, Brussels, 1956.

6. Van Ingen, J. L., "A Suggested Semi-Empirical Method for the Calculation of the Boundary Layer Transition Region," University of Technology, Department of Aero. Engineering Report UTH-74, 1956, Delft.

7. Malik, M. R., 1990, "Numerical Methods for Hypersonic Boundary Layer Stability," J. Computational Physics, Vol. 86, No. 2, pp. 376-413.

8. L. M. Mack, "Boundary Layer Linear Stability Theory," AGARD Report No. 709, 1984.

9. L. M. Mack, "Three-dimensional effects in boundary-layer stability," Twelfth Symposium on Naval Hydrodynamics, Washington, D.C. 1978.

10. Malik, M. R., Li, F. \& Chang, C.-L. 1994, "Crossflow disturbances in threedimensional boundary layers: nonlinear development, wave interaction and secondary instability," J. Fluid Mechanics, Vol. 268, pp. 1-36. 


\section{Results}

\subsection{Blasius flow}

As a first case we compute the optimum suction distribution for the boundary layer over a flat plate at zero pressure gradient. For the details about the stability theory, the reader is referred to the article by Mack (1984). In all the computations we assign the non-dimensional quantity $x_{m i n}=1$. The Reynolds number at the non-dimensional distance $x=1$ is taken as 600 which is well upstream of the neutral point for the most amplified frequency. The $\mathrm{N}$-factor at the transition location is taken as $N_{o}=9$.

The non-dimensional suction velocity and the growth rate $\sigma$ are defined by

$$
\begin{gathered}
\bar{V}_{o}=\frac{V_{o}}{U_{\infty}} \sqrt{\frac{U_{\infty} L}{\nu}}, \\
\sigma=-\alpha_{i}^{*} L
\end{gathered}
$$

where $U_{\infty}$ is the free-stream velocity, $L$ is the length scale and $-\alpha_{i}^{*}$ is the dimensional imaginary part of the eigenvalue $\alpha$. Figure 1 shows the optimum suction distribution and the corresponding growth rate $\sigma$ for various suction parameter $Q$. The horizontal axis shows the non-dimensional coordinate $x$ and the right hand vertical axis shows the suction velocity. In figure 2 we plotted the $\mathrm{N}$-factor variation at the optimum suction distribution and figure 3 illustrates the convergence history towards the optimum solution. These results are obtained with the polynomial expansions for the $V_{0}$ with 8 polynomials.

Our initial suction distribution "iteration 1 " is concentrated beyond the transition point. It is interesting to observe that within one iteration the suction is redistributed beneath the unstable region up to the transition location $x_{\dot{T}}$, and beyond the transition point the suction is approximately zero. The convergence becomes slower if we have to optimize over the frequency and the spanwise wavenumber. Overall the converged results are obtained within 4 to 5 iteration cycles.

From figures 1 and 2 it is seen that the suction distribution peaks upstream of the maximum growth rate and remains flat in the most unstable region and approaches zero steeply near the transition point. The figures also show that in the maximum growth region the suction becomes smaller than that in the less unstable region. The numerical results of Reed and Nayfeh (1986) showed from their linear triple-deck theory and the computations that the multiplying constant becomes maximum near the lower and upper branch neutral points. If we used the constrint that the square of the total suction is constant the suction distribution should peak near the lower and upper branch neutral points which agrees with our computation.

Figure 4 shows the effect of changing the number of polynomials that we used to represent the suction distribution. The results are presented for $\mathrm{N}=4,6$ and 8 and it is seen that there is not much difference in the distribution between $\mathrm{N}=6$ and 8 . In figure 5 we compared the results that obtained with the polynomial and the sine series expansions for the suction. For the sine series expansions we kept 8 and 12 terms. The suction 
distribution obtained with the sine series expansion has some small oscillations, but the oscillation decreases with increasing number of terms. However the growth rate, $\mathrm{N}$ factor, the transition point and the shape of the suction distribution do not differ between the two representations.

Figure 6 shows the largest transition Reynolds number $R e_{T}$ and the corresponding most amplified frequency $\mathrm{F}$ as a function of the control parameter $Q$. In essence this figure depicts the longest transition region that is possible using the steady suction under this constraint for the Blasius boundary layer. Similar graphs can be obtained for other problems using this method.

\subsection{Crossflow Instability}

As a second example we performed the computations for three-dimensional boundary layers. We considered flow over a swept wedge as the model problem. The inviscid velocity along the axial and the spanwise directions are

$$
\begin{aligned}
& U_{e}=C x^{m}, \\
& W=W_{\infty}=\text { const. }
\end{aligned}
$$

The details about the linear stability for this flow are given in Mack (1978) and Malik et.al (1992). The Reynolds number at $x_{\min }=1$ is

$$
R e_{o}=\frac{L}{\sqrt{\nu / c}}=80,
$$

and the Reynolds number based on the spanwise velocity is

$$
\overline{R e}_{o}=W_{\infty} \frac{L}{\sqrt{\nu / c}}=500 .
$$

Figure 7 shows the optimum suction distribution and the corresponding growth rate $\sigma$ for various suction parameter $\mathrm{Q}$ for the travelling disturbances. In Figure 8 we plot the Nfactor variation at the optimum suction distribution and figure 9 shows the convergence history. The transition point $\mathrm{x}_{\mathrm{T}}$ is defined by $\mathrm{N}_{0}=10$. The conclusions are similar to that of the Blasius boundary layer case. In the crossflow case most of the suction is distributed near the maximum amplification region and in the two-dimensional case the suction distribution is almost flat for most of the region.

For the travelling disturbances the optimization is done for the frequency and the spanwise wavenumber and from the figure 9 we see that the converged results are obtained in 4-5 iteration cycle.

Figures 10-12 show the results for the stationary disturbances. Figure 12 shows the effect of changing the number of polynomials that we used to represent the suction distribution. The results are presented for $\mathrm{N}=8$ and 10 . It is seen that the small oscillations which appear in the distribution decreases with increasing $\mathrm{N}$, but the shape and the transition point do not change. In Figure 13, we compare the growth rate and 


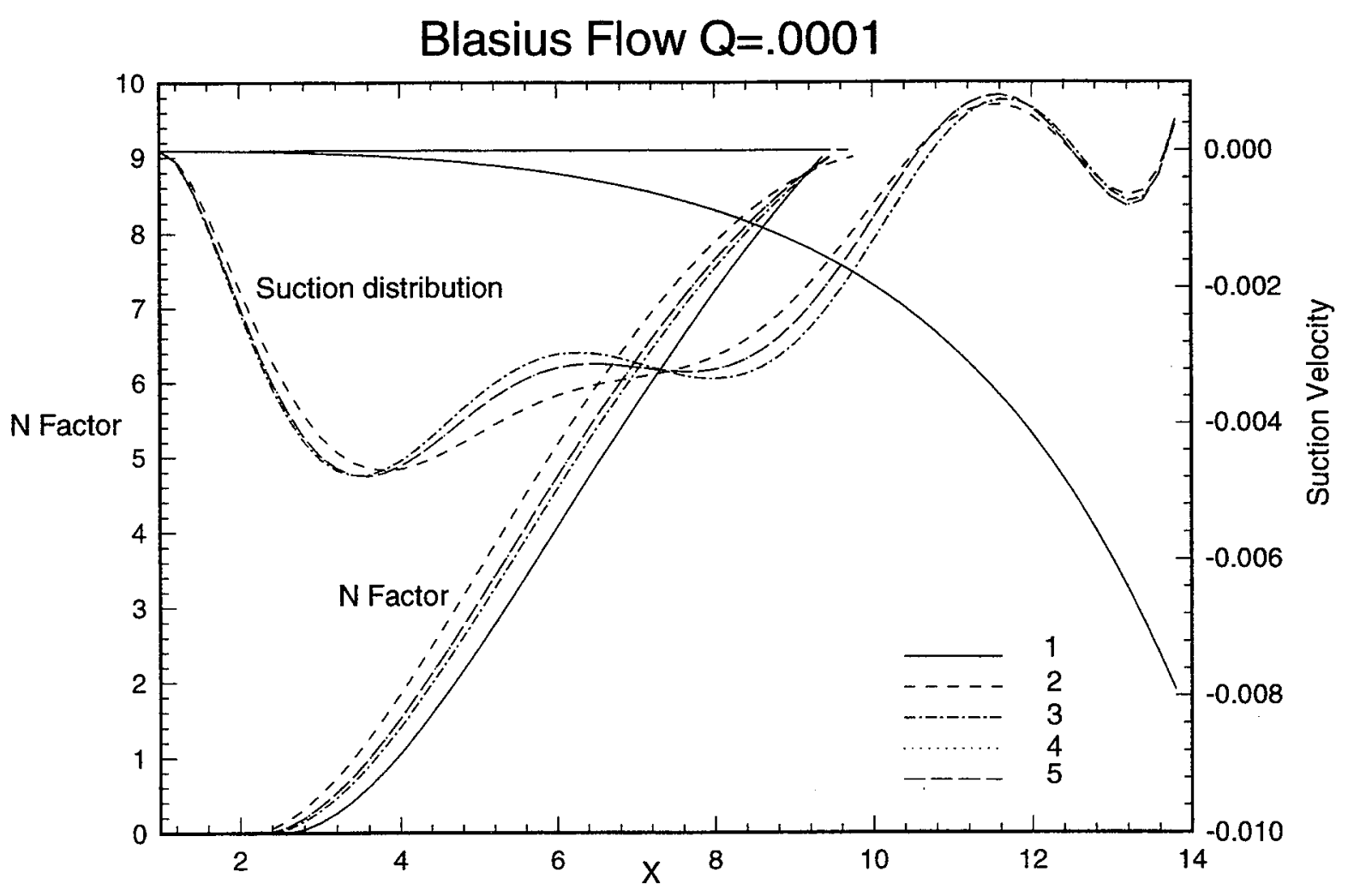

Figure 3. Convergence towards the optimum suction distribution and the growth rate for the Blasius boundary layer $\mathrm{Q}=.0001, \mathrm{~N}=8$. 


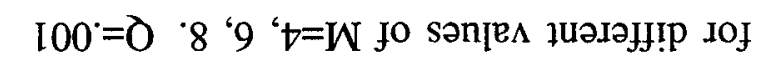

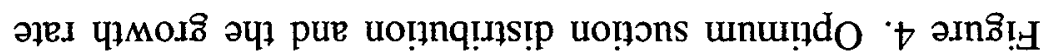

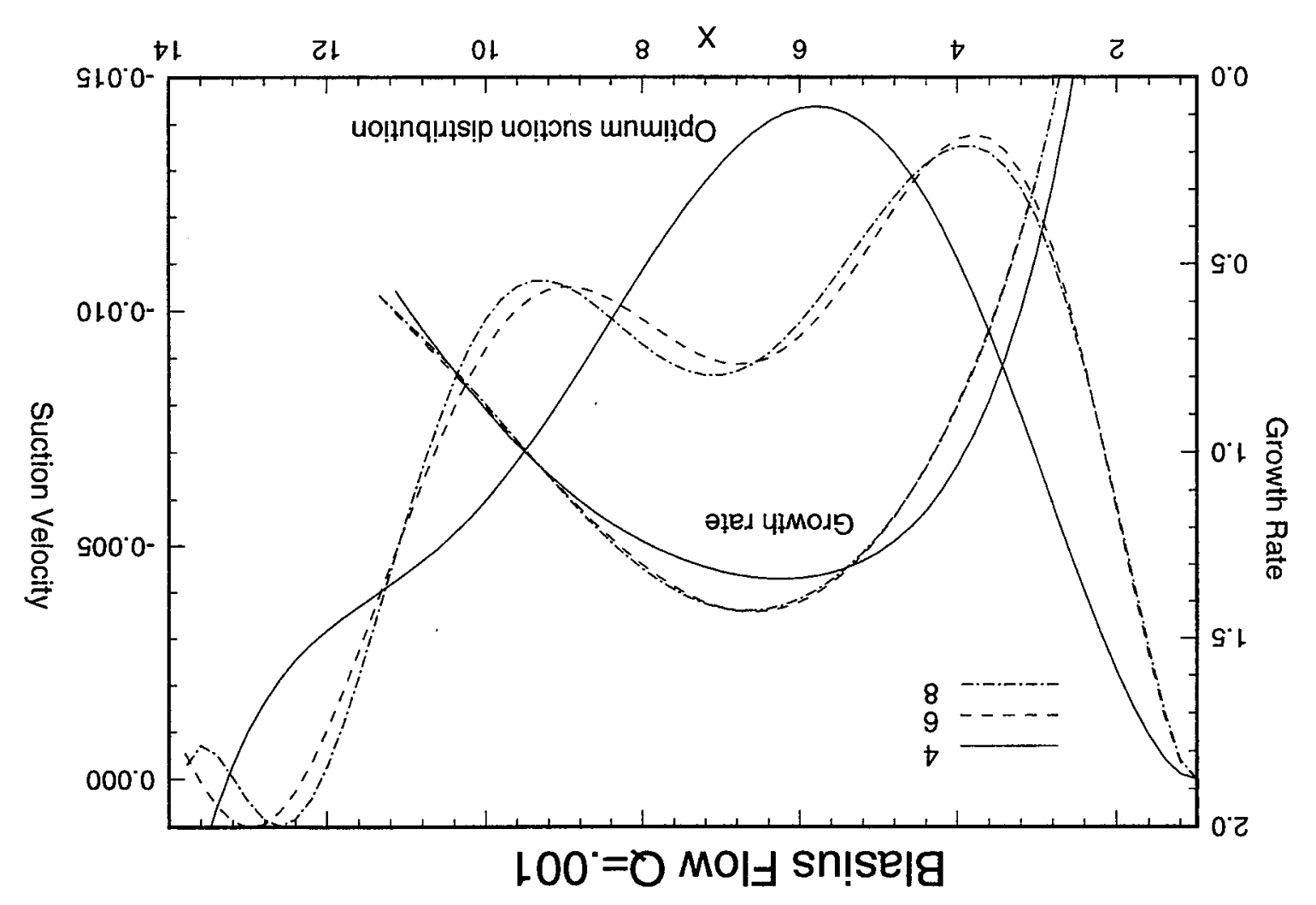




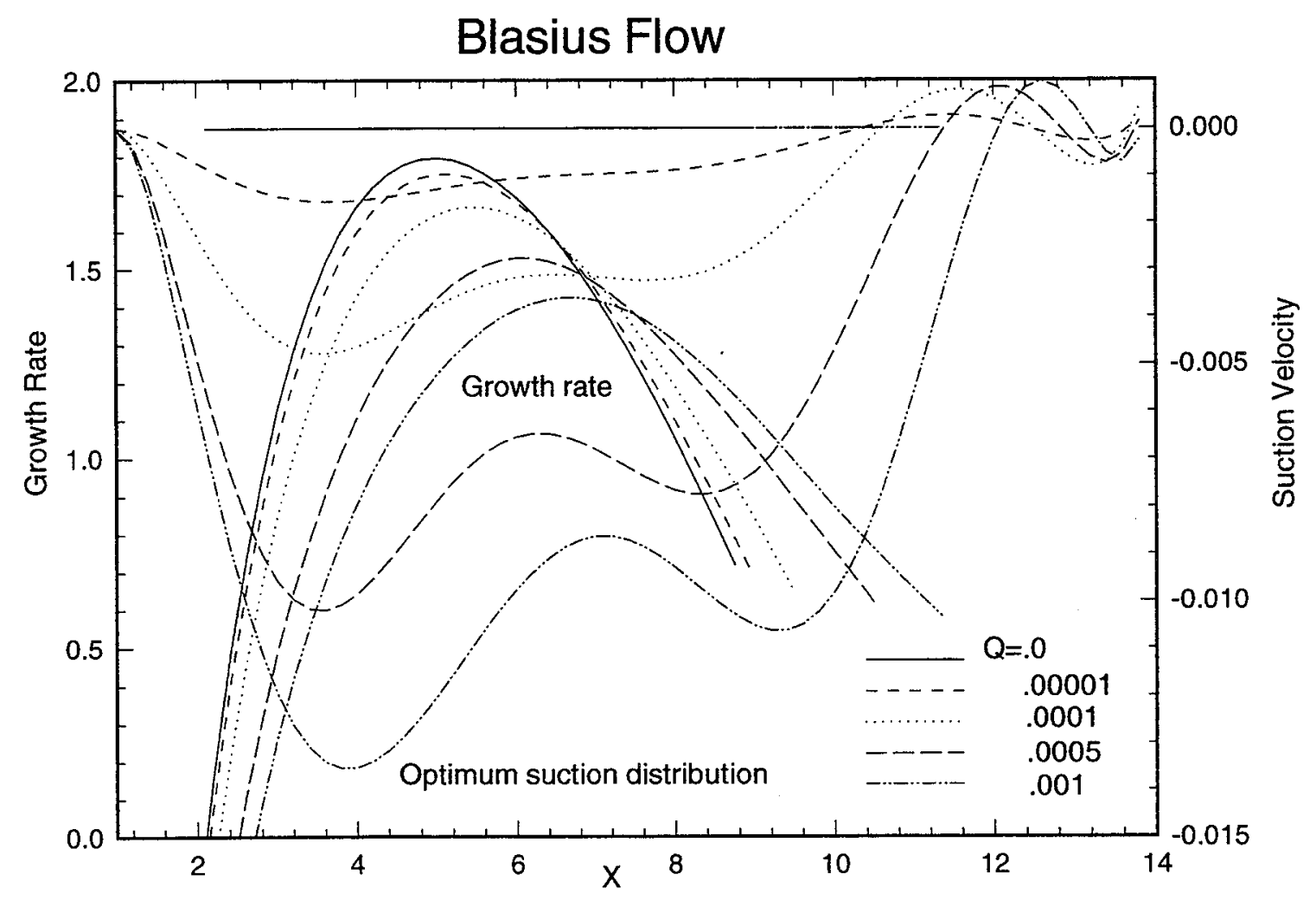

Figure 1. Optimum suction distribution and the growth rate for the Blasius boundary layer. $\mathrm{N}=8$. 


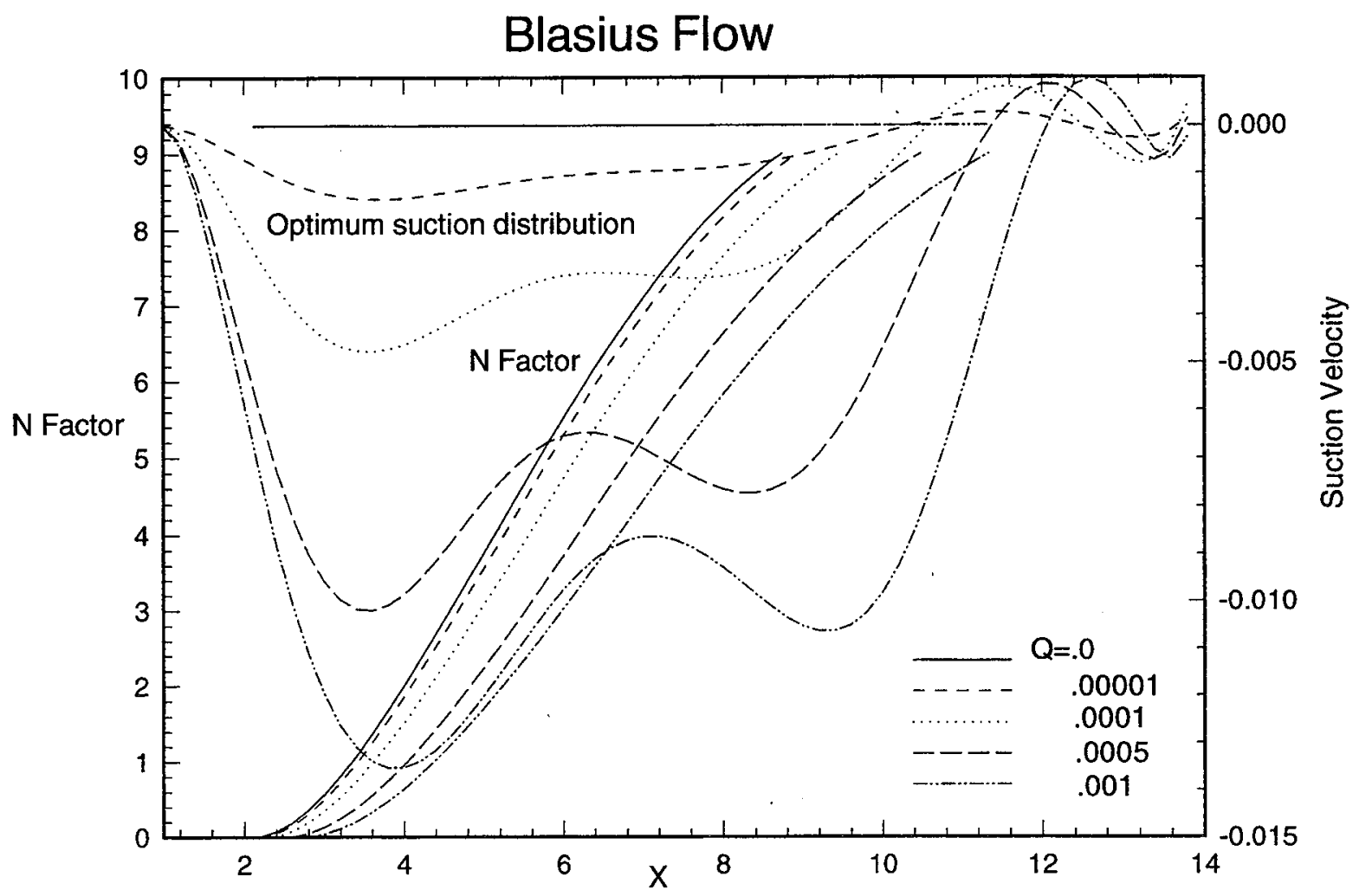

Figure 2. Optimum suction distribution and the $\mathrm{N}$-factor for the Blasius boundary layer. $\mathrm{N}=8$. 


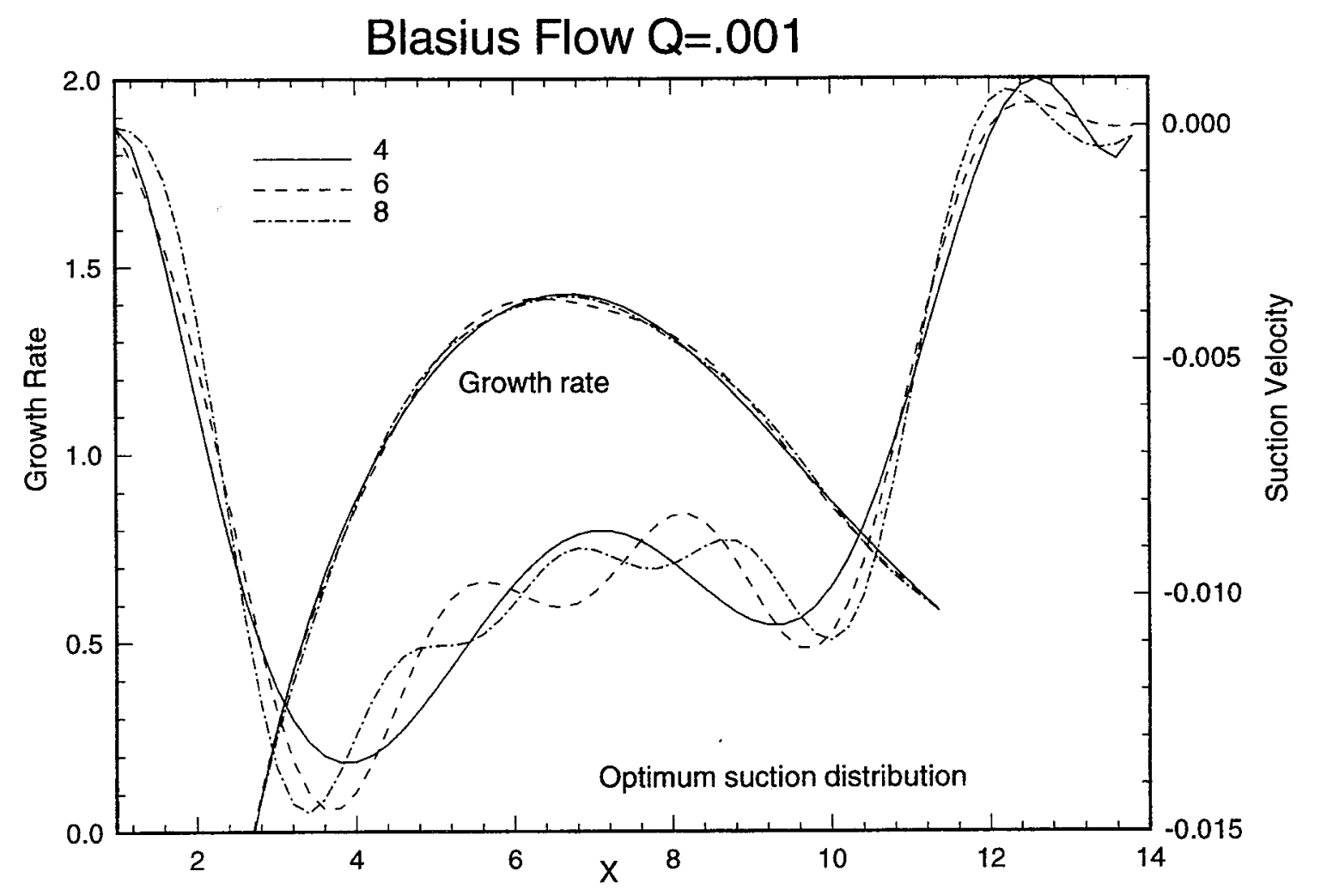

Figure 5. Optimum suction distribution and the growth rate using the polynomial and sine representation for the suction. 


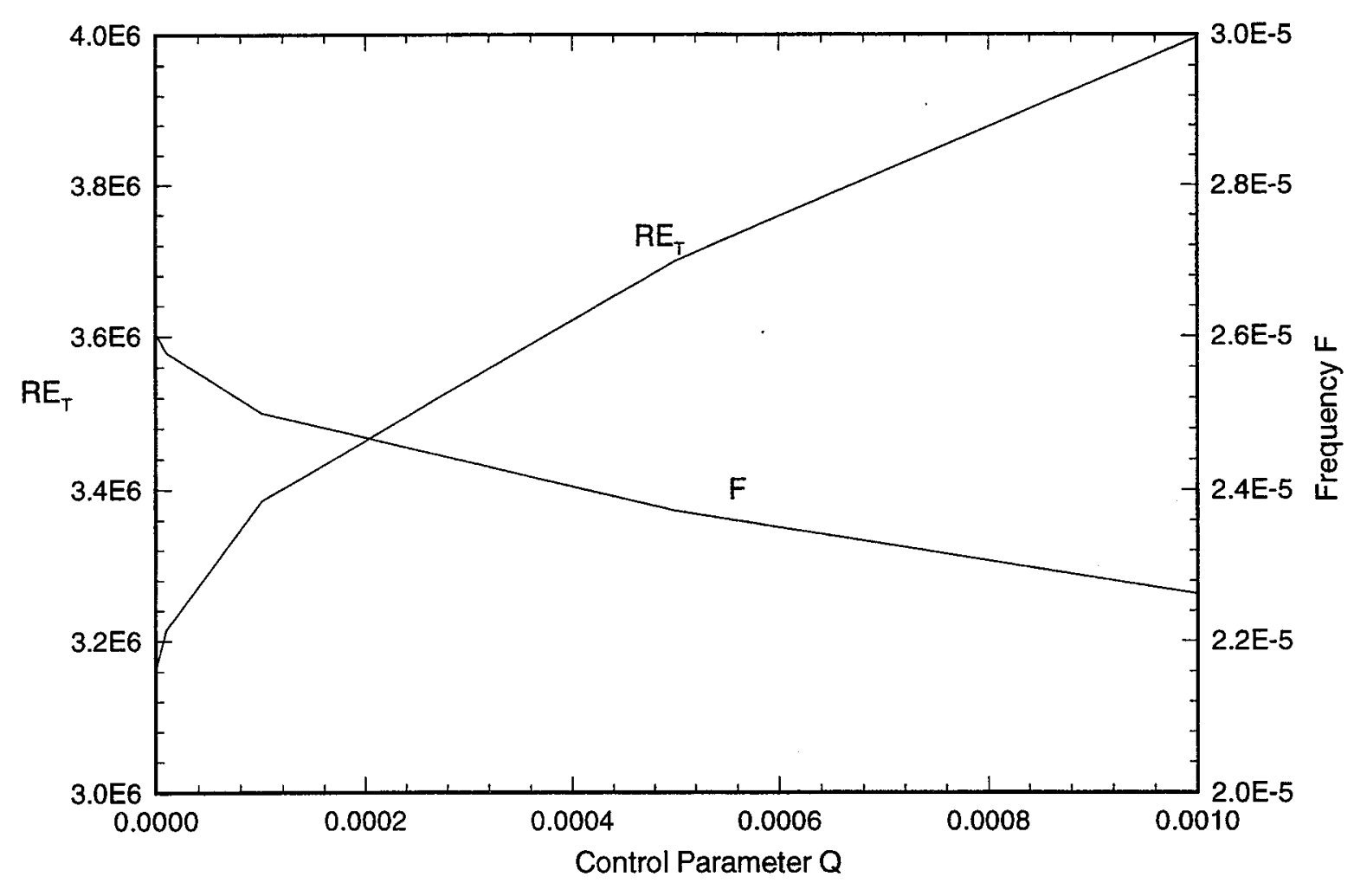

Figure 6. Variation of the maximum transition Reynolds number and the frequency with the suction parameter $Q$. $N=8$. 


\section{Swept Hiemenz Flow Travelling}

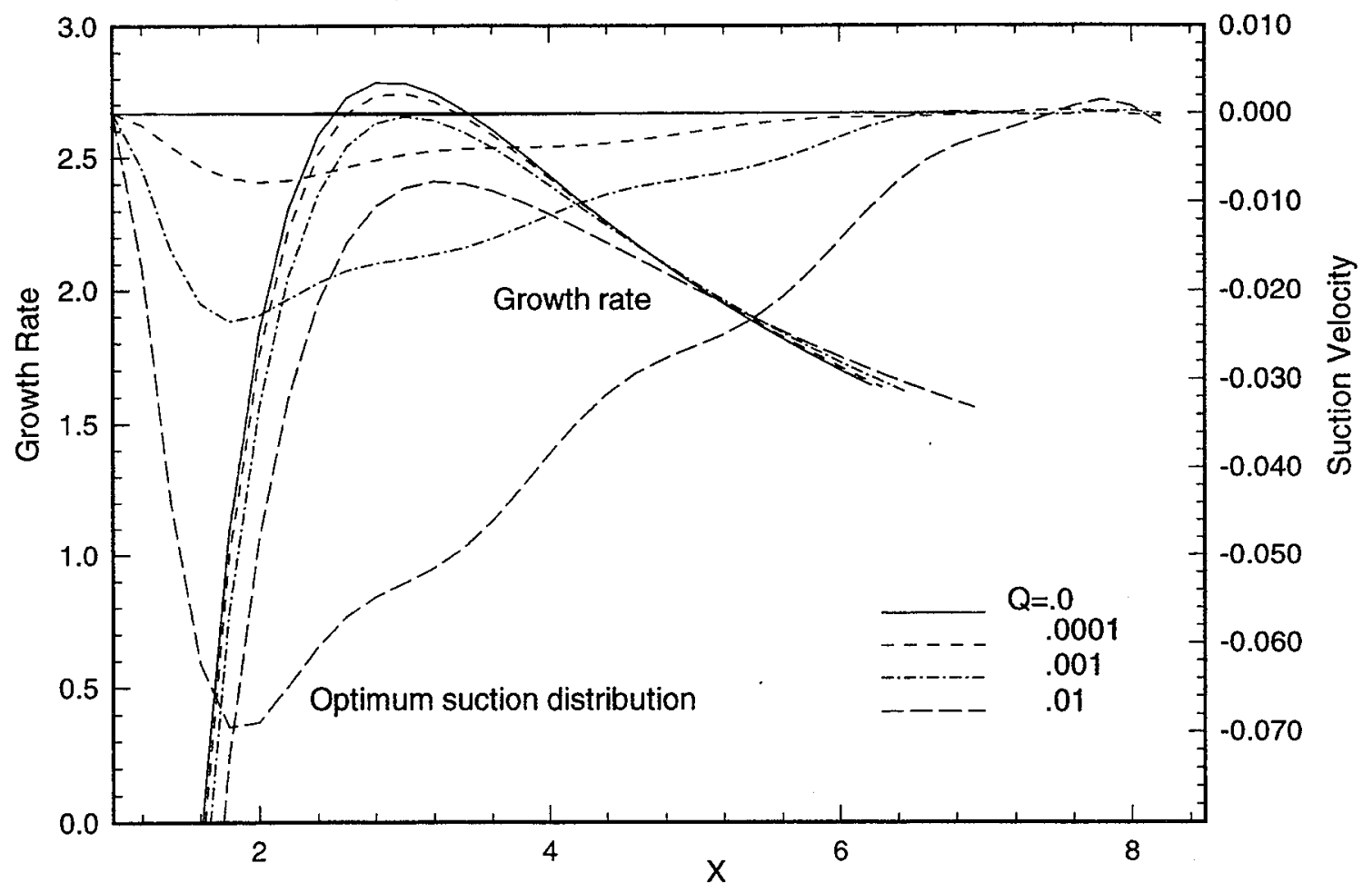

Figure 7. Optimum suction distribution and the growth rate for the swept Hiemenz flow for travelling disturbances. $\bar{R}=500, \mathrm{~N}=8$. 


\section{Swept Hiemenz Flow Travelling}

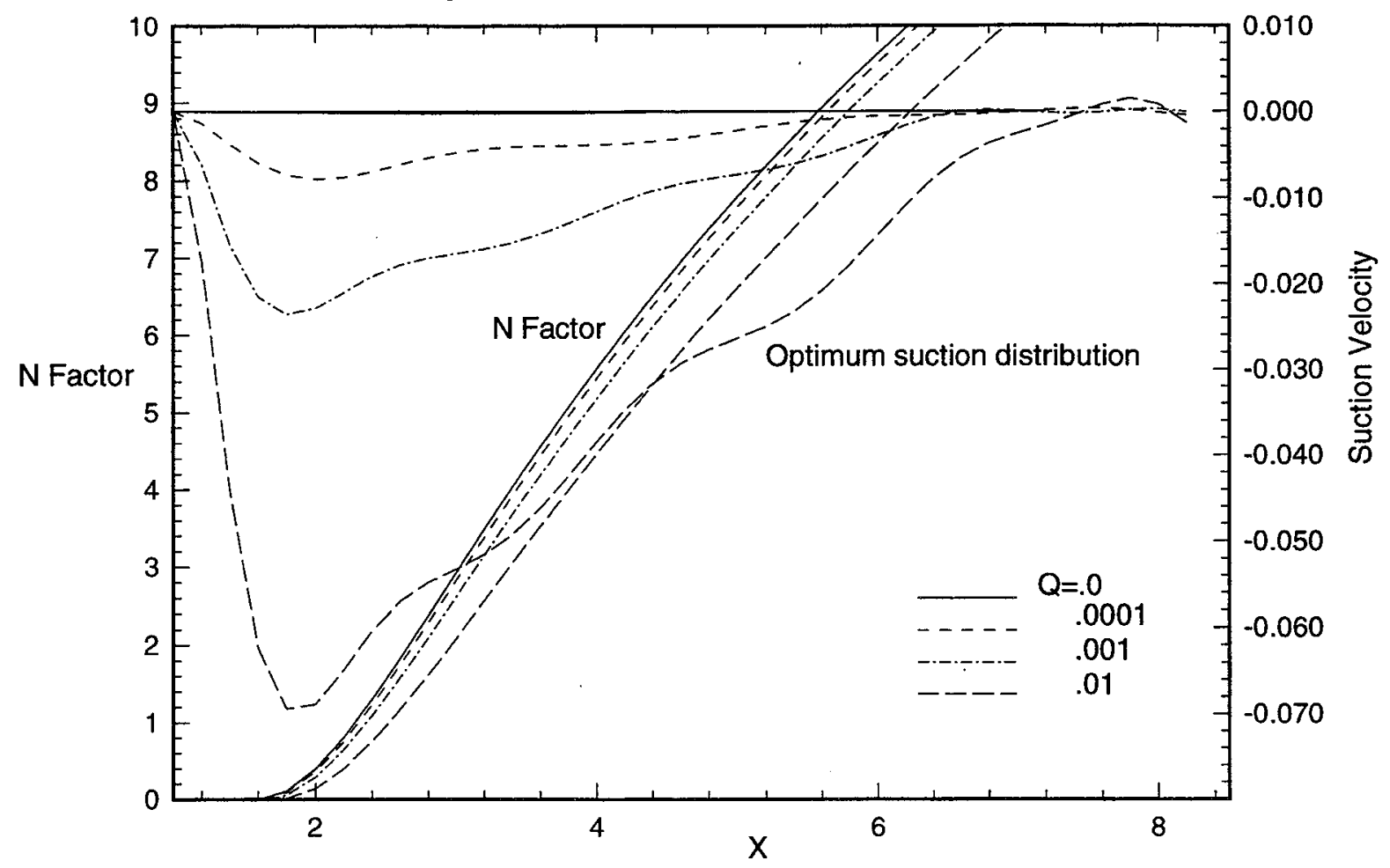

Figure 8. Optimum suction distribution and the $\mathrm{N}$-factor for the swept Hiemenz flow for travelling disturbances. $\bar{R}=500, \dot{\mathrm{N}}=8$. 
Swept Hiemenz Flow Travelling

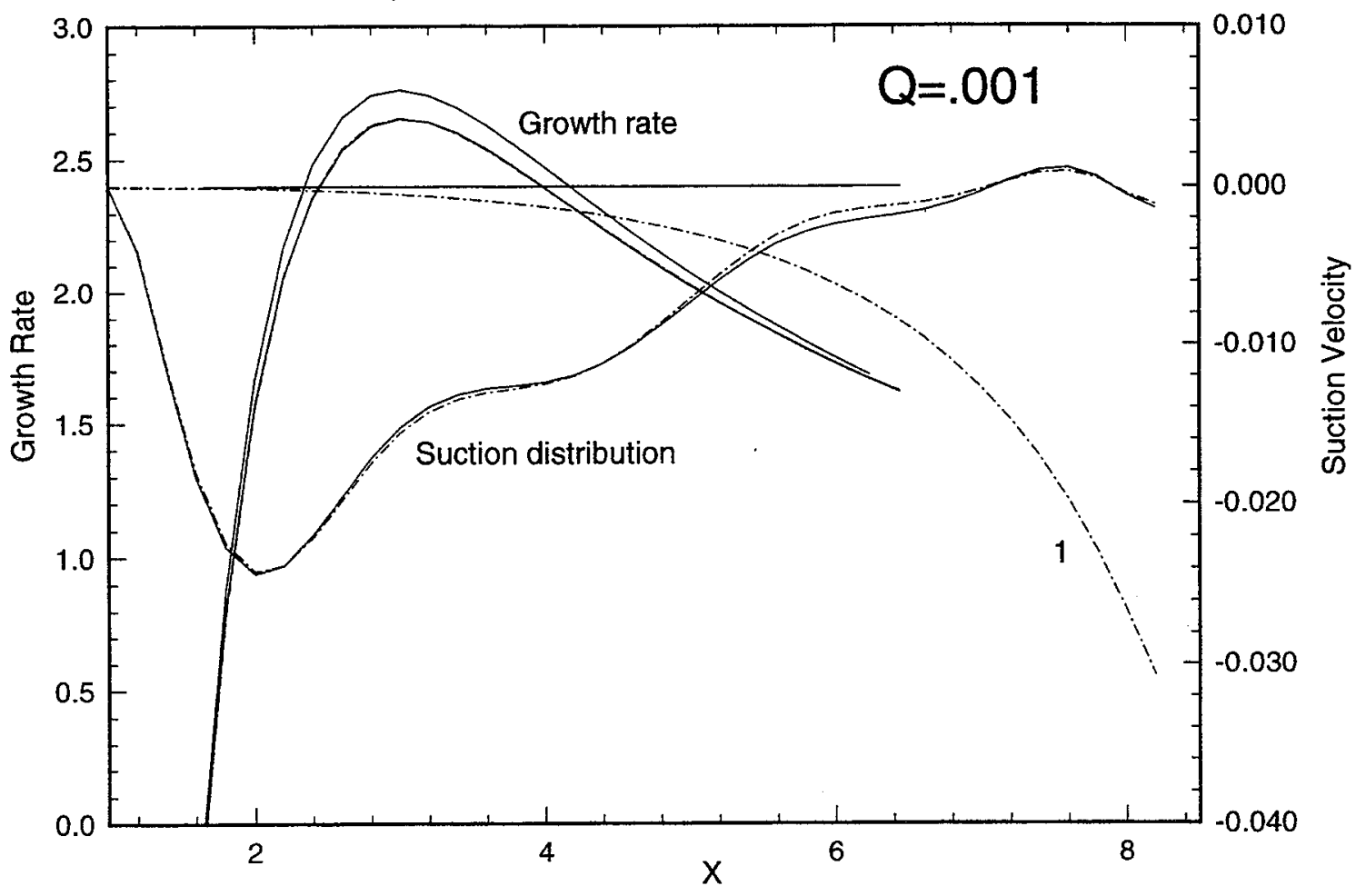

Figure 9. Convergence towards the optimum suction distribution and the growth rate for the swept Hiemenz flow. $\bar{R}=500, \mathrm{Q}=.001, \mathrm{~N}=8$. 


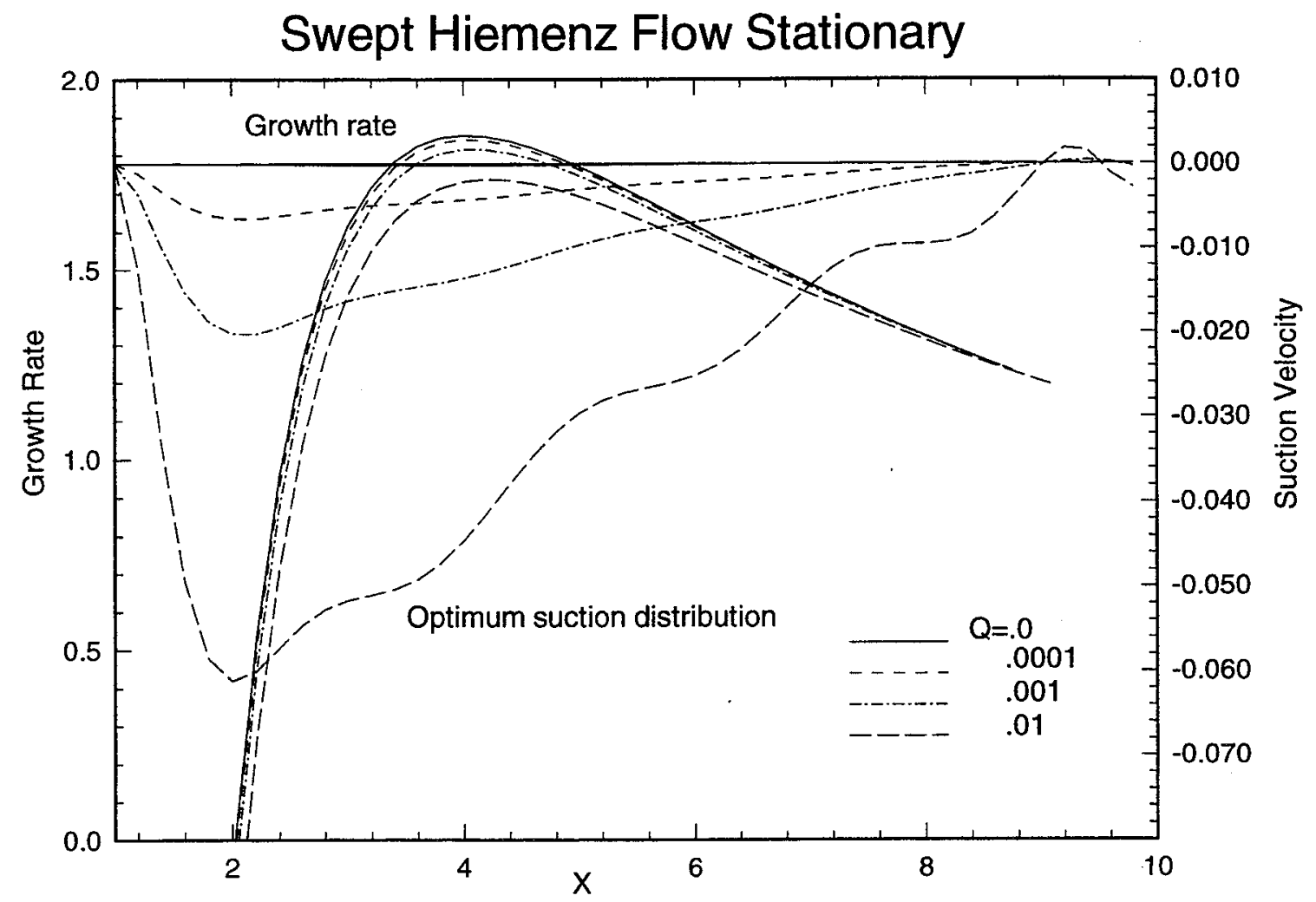

Figure 10. Optimum suction distribution and the growth rate for the swept Hiemenz flow for stationary disturbances. $\bar{R}=500, \mathrm{~N}=10$. 


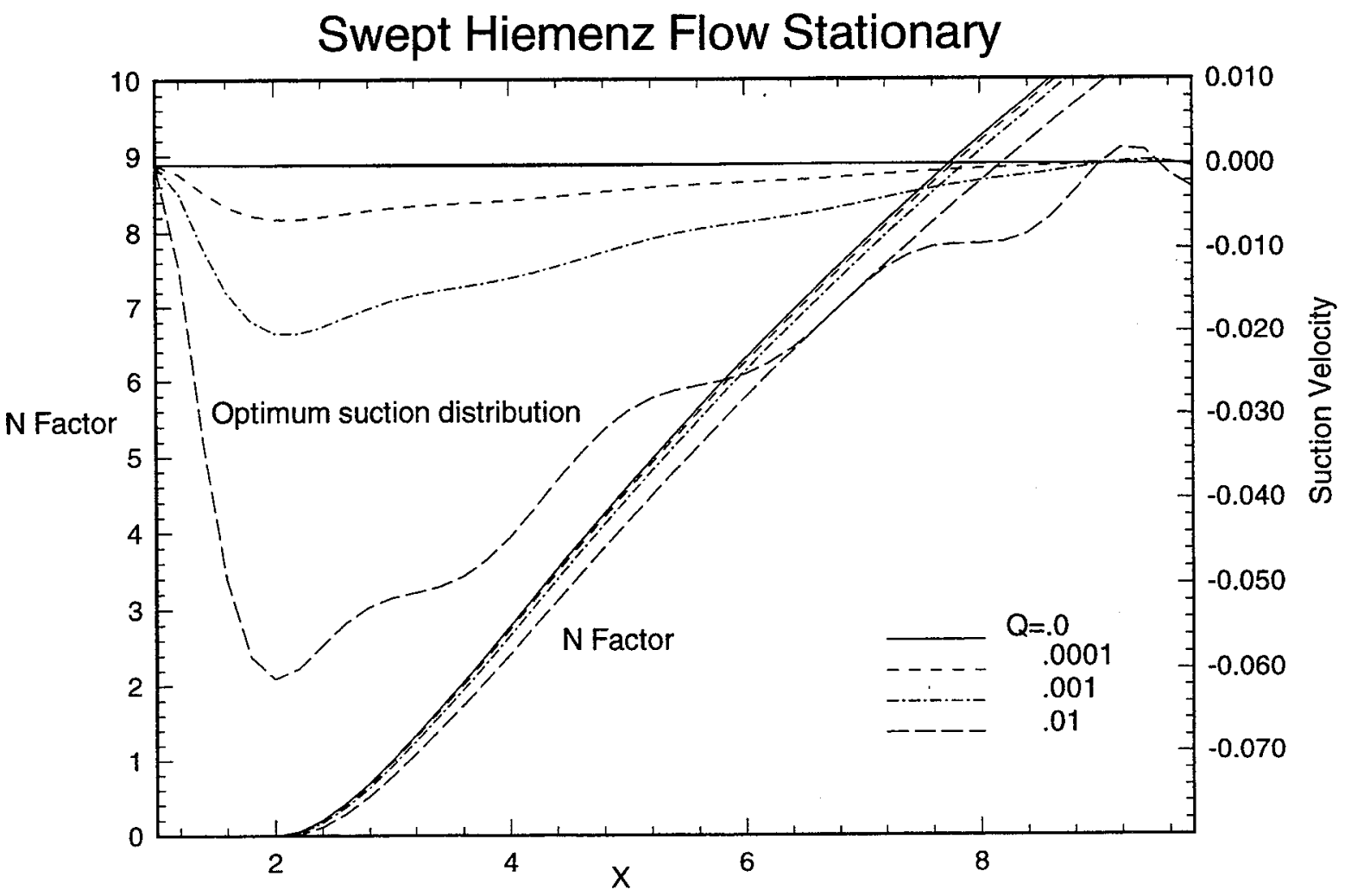

Figure 11. Optimum suction distribution and the $\mathrm{N}$-factor for the swept Hiemenz flow for stationary disturbances. $\bar{R}=500, \mathrm{~N}=10$. 


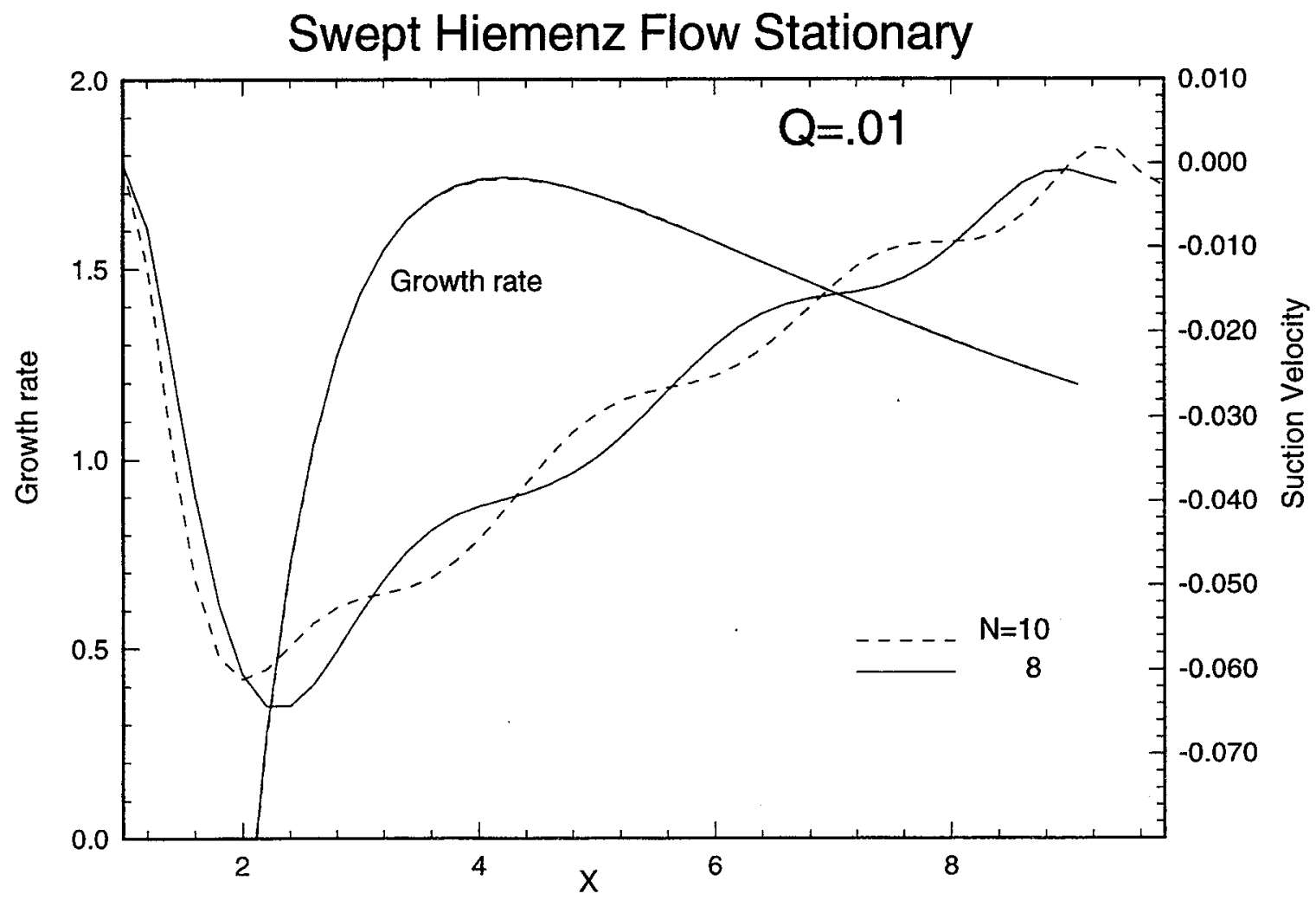

Figure 12. Optimum suction distribution and the $\mathrm{N}$-factor for different values of $\mathrm{N}=8,10$ for $\mathrm{Q}=.01$. 
Swept Hiemenz Flow

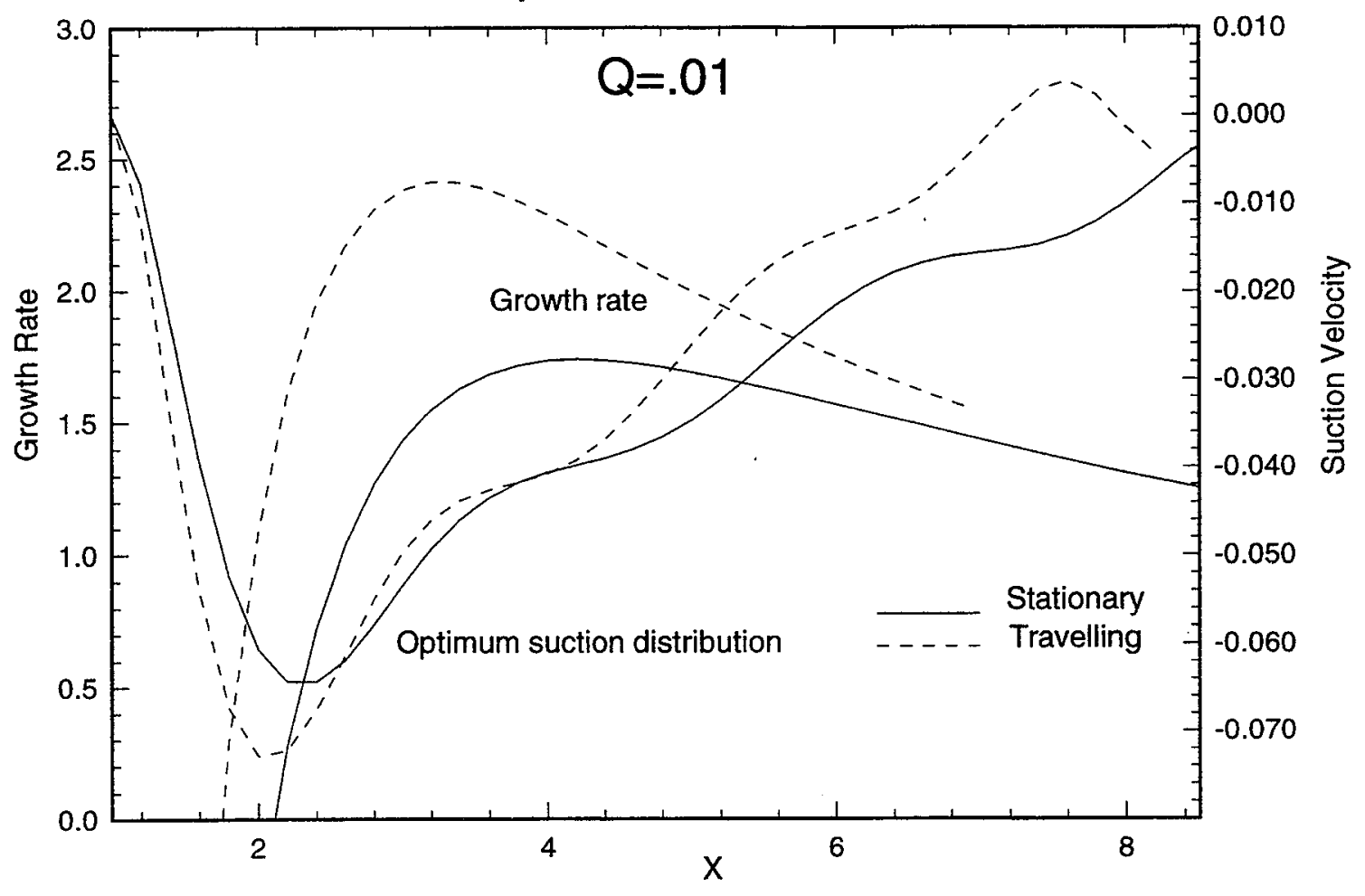

Figure 13. Optimum suction distribution and the $\mathrm{N}$-factor for the stationary and travelling disturbances. $\mathrm{Q}=.01, \mathrm{~N}=8$. 


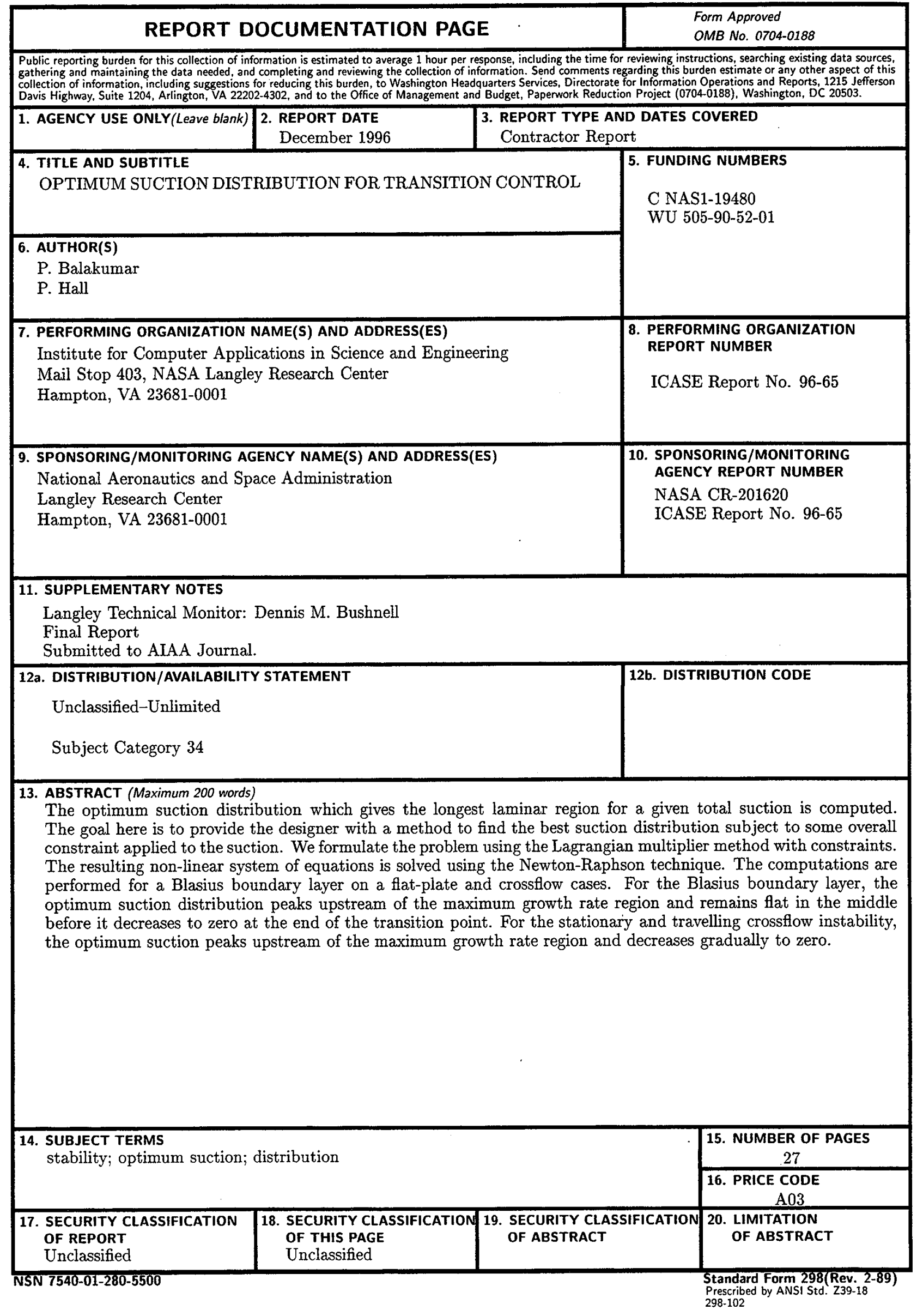

